\title{
Chronische Prurigo: eine ungewöhnliche Manifestation des Hodgkin-Lymphoms
}

\author{
Shireen Dumont ${ }^{a} \quad$ Marc Péchère $^{b} \quad$ Laurence Toutous Trellu $^{b}$ \\ a Internal Medicine Department, Hôpital de la Tour, Geneva, Switzerland; \\ ${ }^{b}$ Department of Dermatology, Geneva University Hospital, Geneva, Switzerland
}

\author{
Schlüsselwörter \\ Prurigo nodularis · Hodgkin-Lymphom · Psychogener Pruritus . \\ Diagnostik
}

\section{Zusammenfassung}

Hintergrund: Prurigo nodularis ist eine Krankheit unbekannten Ursprungs, die durch papulonoduläre Effloreszenzen und starken Juckreiz charakterisiert ist. Hodgkin-Lymphome manifestieren sich anfänglich oft mit unspezifischen Symptomen. Eine Assoziation zwischen systemischen malignen Erkrankungen und kutanen Manifestationen ist schon seit Langem dokumentiert. Wir berichten hier über einen Fall von Prurigo nodularis als Erstmanifestation eines Hodgkin-Lymphoms.

Fall: Eine 35-jährige Frau stellte sich mit seit 2 Jahren bestehendem Pruritus vor. Nachdem zunächst Wanzen als Ursache diagnostiziert wurden, der Pruritus jedoch auch nach Bekämpfung der Insekten anhielt, kamen papulonoduläre Läsionen hinzu, die dem Bild einer chronischen Prurigo entsprachen. Juckreiz und Schmerzen sprachen auf keine Behandlung an. Die Patientin hatte keine einschlägige Vorgeschichte bzw. lagen auch keine früheren klinischen und radiologischen Untersuchungsergebnisse oder Laborbefunde vor. Eine Lymphadenopathie wurde 2 Jahre nach dem Auftreten der ersten Symptome entdeckt. Eine Lymphknotenbiopsie ergab ein nodulär-sklerosierendes Hodgkin-Lymphom. Eine Chemotherapie wurde eingeleitet, und die Hautläsionen gingen zurück.

Schlussfolgerung: Der hier beschriebene Fall einer chronischen Prurigo als erstem Anzeichen einer systemischen malignen Erkrankung unterstreicht, wie wichtig es ist, bei diesen Patienten sowohl anfangs als auch im gesamten Verlauf bei der Diagnose systematisch vorzugehen, insbesondere wenn durch die Behandlung keine Besserung der Beschwerden eintritt. Unser Fall könnte die Rolle der Bildgebung im Management und Follow-up der persistierenden Prurigo nodularis in Frage stellen.

(c) 2018 The Author(s). Veröffentlicht von S. Karger AG, Basel

\section{Hintergrund}

Prurigo nodularis (PN) ist definiert als Vorliegen zahlreicher, symmetrisch verteilter, hyperkeratotischer oder erosiver Knoten insbesondere in Regionen, die zum Kratzen gut erreichbar sind. Das vorherrschende Symptom ist starker Juckreiz $[1,2]$. Ätiologisch kann sie mit vielen anderen Krankheiten in Verbindung stehen, von dermatologischen (atopische Dermatitis, mykobakterielle Hautinfektionen) und systemischen Erkrankungen (HIV-
Infektionen, Anämie, Diabetes, Helicobacter-pylori-Infektionen) bis hin zu psychischen Störungen (Depression, Angst) [3]. Eine direkte Assoziation mit Pruritus ist nicht immer zu erkennen, und die Zeit bis zur Diagnosestellung kann lang sein [4]. PN ist ein bekanntes mögliches Zeichen einer malignen Erkrankung, insbesondere einer lymphoproliferativen Malignität wie Leukämie oder Morbus Hodgkin [3, 5]. Hodgkin-Lymphome (HL) machen rund $10 \%$ aller Lymphome aus, und auch hier ist der Weg zur Diagnose oft langwierig und hindernisreich. Howell et al. [6]

\section{KARGER \\ Fax +4976145207 14 \\ information@karger.com}

www.karger.com

\section{(c) 2018 S. Karger GmbH, Freiburg}

Accessible online at:

www.karger.com/kkd
Laurence Toutous Trellu

Department of Dermatology, Geneva University Hospita

Rue Gabrielle-Perret-Gentil 4

1205 Geneva (Switzerland)

laurence.trellu@hcuge.ch 
berichten von einer Zeitspanne von 87 Tagen zwischen der Erstkonsultation und der HL-Diagnose und von 158 Tagen zwischen der Erstmanifestation von Symptomen und der HL-Diagnose. Wir berichten hier vom Fall einer Patientin mit befundfreier Vorgeschichte, die sich mit PN als isoliertem Prodromalsymptom eines Morbus Hodgkin vorstellte.

\section{Vorstellung des Falls}

Eine 35-jährige Frau klagte bei der Vorstellung über 2 Jahre bestehenden starken Juckreiz in Verbindung mit Schmerzen sowie 14 Monate nach diesen Symptomen aufgetretene papulonoduläre Läsionen (Abb. 1). Die Patientin hatte keine einschlägige Vorgeschichte und erhielt aktuell keine Behandlung. Zunächst waren bei der Patientin zu Hause Wanzen gefunden und durch ein professionelles Schädlingsbekämpfungsunternehmen vernichtet worden, doch auch danach hielt der Juckreiz an. Anfänglich zeigten die Laborwerte lediglich eine erhöhte Immunglobulin E(IgE)Konzentration (272 kU/l; Normal: $<100 \mathrm{kU} / \mathrm{l})$, und auch die klinische Untersuchung und Thoraxröntgen ergaben keine weiteren auffälligen Befunde. Die Patientin konsultierte ihren Gynäkologen, der keine Auffälligkeiten fand. Behandlungen mit Antihistaminika, Phototherapie, topischen Kortikoiden und trizyklischen Antidepressiva brachten keine Symptomlinderung. Lediglich Ganzkörper-Kryotherapiesitzungen bewirkten eine geringfügige Besserung des Pruritus.

Etwa 14 Monate nach dem Einsetzen der ersten Symptome, begannen bei der Patientin papulonoduläre Hautläsionen aufzutreten. Eine Hautbiopsie ergab Hyperkeratose mit epidermaler Akanthose, fokaler Hypergranulose und mononukleärer Infiltration der oberen Dermisschicht; dies entsprach dem Bild einer chronischen PN. Mit Epikutantests wurde nach kutanen Allergien gesucht, doch die Ergebnisse erklärten die extensive Dermatose der Patientin nicht.

Sechs Monate später besuchte sie erneut ihren Hausarzt mit hartnäckigem Juckreiz, Schüttelfrost und quälendem Schmerz. Fieber, Nachtschweiß oder Gewichtsverlust lagen in der Anamnese nicht vor, jedoch klagte die Patientin über starke Müdigkeit. In der körperlichen Untersuchung zeigten sich feste, konturierte Hautknoten von 0,3-2 cm Größe an den Streckseiten der Extremitäten, am Rücken und am Gesäß. Exkoriationen und hyperchrome Vernarbungen früherer Läsionen lagen ebenfalls vor. Eine klinische Untersuchung ergab keine Hinweise auf Adenopathie oder Hepatosplenomegalie.

Eine Behandlung mit Dexamethason $6 \mathrm{mg} /$ Tag wurde eingeleitet. Nach der Anwendung des Kortikosteroids verschlechterten sich die Symptome, und eine palpable supraklavikuläre Lymphadenopathie trat auf. Im Thoraxröntgen zeigte sich eine mediastinalparatracheale Raumforderung. Ein CT-Scan bestätigte diesen Befund und zeigte außerdem mehrere anterior-mediastinale, rechts-paratracheale und rechts-hiläre Lymphadenopathien.

Die Laboruntersuchungen erbrachten folgende Ergebnisse: IgEKonzentration 130,0 kU/l (Normbereich $<100$ ), C-reaktives Protein 13,38 mg/l (Normbereich <5), Leukozyten 11,9 G/1 (Norm-

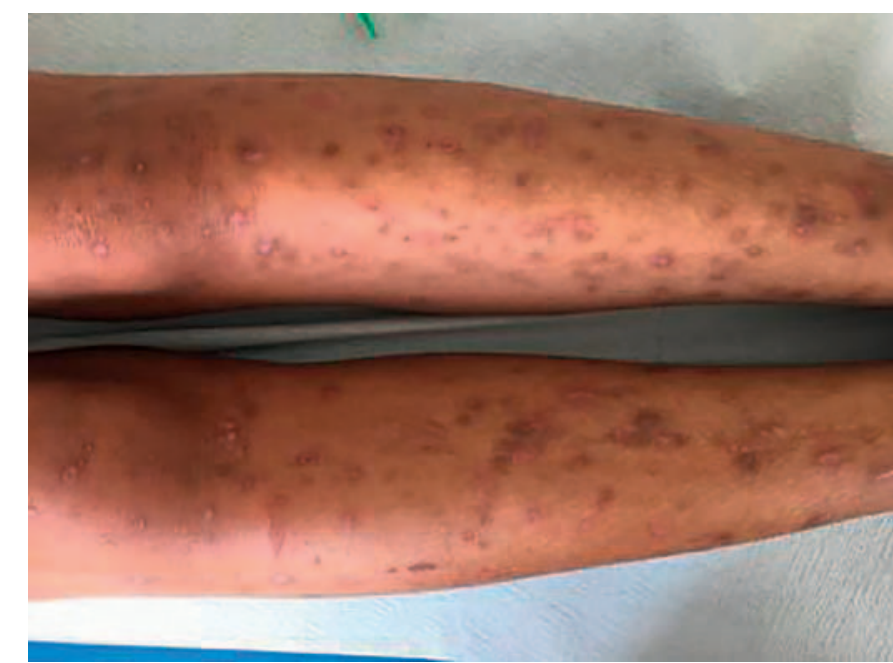

Abb. 1 Prurigo-Papeln mit Kratzspuren und Pigmentierung an den Beinen.

bereich 4-10), Immunglobulin M 3,71 (Normbereich 0,40-2,30). Eine Proteinelektrophorese blieb ohne Befund. Hepatitis-, HIV-, Toxoplasmose-, Parvovirus-B19-, Syphilis-, Epstein-Barr-Virus-, Bartonellose-, Rickettsiose-, Q-Fieber-, Brucellose- und Tuberkulose-Infektionen wurden mittels serologischer Tests ausgeschlossen. Eine Lymphknotenbiopsie ergab ein polymorphes lymphoides Infiltrat mit fibrösen Bändern sowie das Vorliegen von Sternberg-Reed-Riesenzellen, neoplastischen Zellen (CD30-, MUM1-, PD-L1- und CD20-positiv; CD15-negativ) und T-Zellen (CD3-positiv). Diese Ergebnisse waren vereinbar mit einem HL vom nodulär-sklerosierenden Typ. Eine Chemotherapie wurde eingeleitet, ausgelegt auf 12-14 Behandlungssitzungen mit Adriamycin, Bleomycin, Vinblastin und Dacarbazin (ABVD). Nach der ersten Sitzung waren Juckreiz und Schmerzen gelindert, und die Hautläsionen nahmen ab.

\section{Diskussion}

Chronischer Juckreiz beeinträchtigt in hohem Maße die Lebensqualität und stellt diagnostisch und therapeutisch eine echte $\mathrm{He}$ rausforderung dar. Unser Fall illustriert die Komplexität der PN, für die eine klare ätiologische Diagnose nicht immer leicht $\mathrm{zu}$ benennen ist. Deshalb wird oft zunächst eine symptomatische Behandlung versucht, die langfristig jedoch enttäuschend verlaufen kann. Unsere Patientin stellte sich mit Juckreiz vor, der auch nach Eradikation von Wanzen und nach wiederholten Untersuchungen durch mehrere Ärzte persistierte. Da keine weiteren relevanten Befunde vorlagen, galten weiterhin die Insekten als unmittelbare Ursache des Juckreizes bzw. als auslösender Faktor für psychische Angstzustände, die zur Verstetigung des Juckreizes führten. So wurde der Pruritus zunächst als psychogen fehldiagnostiziert, bis schließlich, 2 Jahre nach der Erstmanifestation der Symptome, die Diagnose HL gestellt wurde. Die gängigen Untersuchungen hatten jedoch keine stimmigen Krankheitszeichen ergeben. 
Unser Fall illustriert die üblichen Schritte der klinischen Versorgung und das Fehlen von Frühwarnzeichen für bestimmte Lymphomarten. Die späte Diagnosestellung hat mehrere negative Auswirkungen, wenn die Beschwerden der Patienten übergangen werden, obwohl der Juckreiz symptomatisch für eine schwere Erkrankung ist. Im Zuge dessen könnten sich Patienten auch vernachlässigt fühlen und das Vertrauen in die Ärzte verlieren, was wiederum zu einer unzureichenden weiteren Versorgung führen könnte. Schon in früheren Publikationen steht, dass eine generalisierte PN ohne primäre Dermatose Untersuchungen zum Ausschluss systemischer Erkrankungen einschließlich von neoplastischem Pruritus erforderlich macht [7]. Selbst bei Verdacht auf einen psychischen Ursprung ohne sonstige erkennbare Ätiologie trotz umfassender Untersuchungen sollten Ärzte ein relativ breites Spektrum von Differenzialdiagnosen im Blick behalten und auf jegliche neuen klinischen Zeichen achten, z.B. das Auftreten einer Adenopathie. Lymphadenopathie ist Berichten zufolge das häufigste klinische Merkmal des HL [8], und kutane paraneoplastische Manifestationen kommen bei $17-53 \%$ aller HL-Patienten vor [9]. Die Rolle bildgebender Untersuchungen in der Diagnostik und Behandlung von Lymphomen ist bereits diskutiert worden [10, 11]; jedoch könnte, auch unter Berücksichtigung von epidemiologischen Faktoren und dem klinischen Kontext, unser Fall ihren Nutzen im Hinblick auf persistierende PN in Frage stellen.

\section{Schlussfolgerungen}

Dieser Fall liefert neue Belege für einen Zusammenhang zwischen PN und HL. Es ist somit wichtig, sich zu vergegenwärtigen, dass chronischer Pruritus immer ein systematisches diagnostisches Vorgehen sowohl zu Beginn als auch im weiteren Verlauf erfordert, insbesondere wenn ein Patient nicht auf eine Behandlung anspricht. Mehrere Aspekte wie die Vorgeschichte des Patienten und epidemiologische Faktoren sollten dazu beitragen, die Rolle bildgebender Untersuchungen in der Diagnostik der PN neu zu definieren.

\section{Erklärung zu ethischen Konflikten}

Von der Patientin wurde nach Aufklärung eine schriftliche Einverständniserklärung für die Veröffentlichung des vorliegenden Fallberichts und der dazugehörigen Bilder eingeholt.

\section{Disclosure Statement}

Die Autoren erklären, dass keine Interessenkonflikte bestehen.

\section{Beiträge der einzelnen Autoren}

M.P. war unmittelbar an der Versorgung der Patientin beteiligt. S.D. erstellte die erste Fassung des Manuskripts und analysierte die Literatur. L.T.T. beaufsichtigte die Manuskripterstellung. Alle Autoren haben die finale Fassung des Manuskripts gelesen und freigegeben.

\section{Literatur}

1 Mettang T, Vonend A, Raap U: Prurigo nodularis bei Dermatosen und systemischen Erkrankungen. Hautarzt 2014;65(8):697-703.

2 Schweda K, Hainz M, Loquai C, Grabbe S, Saloga J, Tuettenberg A: Prurigo nodularis as index symptom of (non-Hodgkin) lymphoma: ultrasound as a helpful diagnostic tool in dermatological disorders of unknown origin. Int J Dermatol 2015;54(4):462-464.

3 Kleyn CE, Lai-Cheong JE, Bell HK: Cutaneous manifestations of internal malignancy: diagnosis and management. Am J Clin Dermatol 2006;7(2):71-84.

4 Iking A, Grundmann S, Chatzigeorgakidis E, Phan NQ, Klein D, Ständer S: Prurigo as a symptom of atopic and non-atopic diseases: aetiological survey in a consecutive cohort of 108 patients: J Eur Acad Dermatol Venereol 2013;27(5):550-557.
5 Setoyama M, Mizoguchi S, Kanzaki T: Prurigo as a clinical prodrome to adult T-cell leukaemia/lymphoma. Br J Dermatol 1998;138(1): 137-140.

6 Howell DA, Smith AG, Jack A, Patmore R, Macleod U, Mironska E, Roman E: Time-to-diagnosis and symptoms of myeloma, lymphomas and leukaemias: a report from the Haematological Malignancy Research Network. BMC Hematol 2013;13(1):9.

7 Tan WS, Tey HL: Extensive prurigo nodularis: characterization and etiology. Dermatology 2014;228(3):276-280.

8 Shephard EA, Neal RD, Rose PW, Walter FM, Hamilton WT: Quantifying the risk of Hodgkin lymphoma in symptomatic primary care patients aged $\geq 40$ years: a case-control study using electronic records. Br J Gen Pract 2015 ;65(634):e289-294.
9 Rubenstein M, Duvic M: Cutaneous manifestations of Hodgkin's disease. Int J Dermatol 2006;45(3):251-256.

10 Barrington SF, Mikhaeel NG: When should FDG-PET be used in the modern management of lymphoma? Br J Haematol 2014;164(3): 315-328.

11 Kostakoglu L, Evens AM: FDG-PET imaging for Hodgkin lymphoma: current use and future applications. Clin Adv Hematol Oncol 2014;12(1):20-35. 